\title{
Primary school children often underestimate their nasal obstruction*
}

\author{
Francesca Occasi, Marzia Duse, Tommaso Vittori, Anna Rugiano, Giancarlo \\ Tancredi, Giovanna De Castro, Luciana Indinnimeo, Anna Maria Zicari
}

Rhinology 54: 164-169, 2016

DOI:10.4193/Rhino15.120

*Received for publication:

April 27, 2015

Accepted: October 26, 2015

Department of Pediatrics, University “La Sapienza”, Rome, Italy

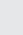

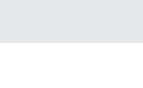

\begin{abstract}
Background: No consensus has ever been reached about the correlation between nasal resistance and the subjective sensation of nasal patency. The aim of the present study was to better define whether primary school and secondary school aged children correctly estimate their nasal obstruction.
\end{abstract}

Materials and methods: Two hundred eighty four children (168 males and 116 female) aged between 6 and 14 years $(9.5+2.9$ years) affected by Pediatric Allergic Rhinitis underwent Rhinomanometry and they were considered as correctly estimating their nasal obstruction when the grade of nasal patency corresponded to the severity of the NOSE score, overestimating when the grade of nasal patency was $<1$ when compared to the severity of the score, underestimating when the grade of nasal patency was $>1$ when compared to the severity of the score.

Results: Correlation between NOSE score and nasal patency was statistically significant $(r-0.74 ; p<0.001)$. Children between 6 and 9 years of age underestimate (43.7\%) and children $>12$ overestimate (34.7\%) their symptoms more frequently than children among other age ranges $(p<0.001)$.

Conclusion: Although NOSE score approximately allow to quantify nasal obstruction, in children, especially between 6 and 9 years of age, an objective measurement of nasal patency should be performed to better define the therapeutic approach.

Key words: allergic rhinitis, nasal obstruction, rhinomanometry, NOSE score, children

\section{Introduction}

Nasal obstruction has been reported as a key symptom of allergic rhinitis since it is related to its well-known impact on children's cognitive performance, school performance, sleep disorders and quality of life ${ }^{(1)}$. Moreover, it is considered the symptom reflecting more directly the allergic inflammation in the nasal mucosa and, hence, a potential trigger of the systemic inflammation underlying allergic rhinitis which is characterized by the release of pro-inflammatory mediators locally as well as in the systemic circulation ${ }^{(2)}$. Nasal blockage can be assessed subjectively with Visual Analogue Scales (VAS) or symptom rating scores and with objective methods, such as active ante- rior rhinomanometry assessing nasal patency quantitatively. Moreover, the reliability of many studies including subjective evaluation of nasal patency may be influenced by the use of not validated nasal symptom scores, even though, also the accuracy of validated questionnaire has never been assessed at different pediatric age ranges. The relation between objective measurements and subjective nasal patency symptoms has been pondered over for decades and no consensus has never been reached about the correlation between nasal resistance and subjective sensation of nasal patency ${ }^{(3)}$. In 2009, André et al. ${ }^{(3)}$ concluded that in the presence of obstructive symptoms a correlation with objective test is more likely to be found and, 
hence, that the subjective sensation alone may reliably describe the 'objective' nasal airway, decreasing the need for objective measurements. However, the authors addressed the lack of a consensus to the point that no validated questionnaires were used in previous studies and they strongly advised the use of validated subjective scoring tools for future studies to enhance the reliability of conclusions concerning the correlation between objective and subjective outcomes. Recently, another group of study concluded that correlations between the subjective and objective techniques were weak and hypothesized that each of these techniques should be regarded as complementary assessing different aspects of nasal obstruction ${ }^{(4)}$.

The subjective perception of nasal patency is mainly due to the activity of specific nasal sensory nerve endings of the major palatine nerve. However, the application of camphor, eucalyptus, L-menthol, vanilla, or lignocaine to the nasal or even palatal mucosa can influence the perception of nasal flow independently from any objectively measurable change in nasal resistance ${ }^{(3)}$. The primary physiological mechanism that produces the sensation of ample nasal airflow is the activation of trigeminal cool thermoreceptors, specifically transient receptor potential melastatin family member 8 (TRPM8), by nasal mucosal cooling ${ }^{(5)}$ while mechanoreceptors do not seem to play a role in sensing nasal airflow. Cool thermoreceptors in the nasal mucosa are connected to respiratory centers and consequently can alter respiration patterns. Moreover, neural hypersensitivity has been observed in patients affected by allergic and non-allergic rhinitis and it is probably related to sodium channel trafficking and characterized by an increase in the number of sensory neurons including nociceptors and immunoreactive nerve fibers of the subepithelium ${ }^{(6)}$. Recently, Thorstensen et al. ${ }^{(7)}$ showed that subjects with asthma often overestimate the sensation of nasal obstruction further arguing the difficulty to solve the conundrum underlying this topic.

In children, a discrepancy between subjective and objective assessment of the degree of nasal blockage has been often observed and schoolchildren on long-term treatment for perennial rhinitis frequently under-report their symptom of nasal stuffiness when compared to healthy controls ${ }^{(8)}$.

Studies focusing specifically on this issue including validated subjective scoring and investigating the perception of nasal patency at different pediatric age groups are lacking. The aim of the present study was to better define whether primary and secondary school aged children correctly estimate their nasal obstruction.

\section{Materials and methods}

Study population

The study was performed at the Allergology and Immunology service of the Pediatric Department, "Sapienza" University of Rome. Two hundred eighty four children (168 males and 116 female) aged between 6 and 14 years (mean age $9.5+2.9$ years) affected by persistent allergic rhinitis (PER), defined as symptoms that occur for more than 4 days/week and for more than 4 consecutive weeks were enrolled ${ }^{(9,10)}$. Atopic status was assessed by a Skin Prick Test (SPT) (Lofarma, Milan, Italy). A positive SPT was defined by the presence of a heal more than $3 \mathrm{~mm}$ respect to the wheal size of control (saline solution). Sensitizations were as follows: $81(28 \%)$ were monosensitized (75 to dust mites and 6 to molds) and 203 (72\%) were polysensitized.

Treatment with topic and systemic antihistamines, corticosteroids was suspended for at least two month before the evaluation and children with nasal abnormalities, concurrent nasal infection, chronic inflammatory disorders such as asthma and inflammatory bowel syndrome, immunodeficiencies, reumathologic, hemathologic and genetic diseases were excluded.

Nose obstruction symptom evaluation score and anterior active rhinomanometry

A nose obstruction symptom evaluation score (NOSE, maximum 100) with a 4-point scale to identify the severity of Nasal congestion or stuffiness, Nasal blockage or obstruction, Trouble breathing through the nose, Trouble sleeping, Unable to get enough air through the nose during exercise or exertion was obtained for each child ${ }^{(11)}$. The questionnaire was completed by one investigator who interviewed the patients. When necessary parents were asked to help the child without guiding or interfering with his responses. According to the NOSE score patients were categorized as having 1-mild (range, 5-30), 2-moderate (range, 30-55), 3-severe (range, 55-80), or 4-very severe (range, 80-100) nasal obstruction (12).

All patients underwent Anterior Active Rhinomanometry (Sibelmed Rinospir PRO 164) in accordance with the International Committee on Standardization of rhinomanometry ${ }^{(13)}$. A retest was performed after 15-30 minutes. The results of rhinomanometry were considered at a fixed transnasal pressure of 150 Pascal $(\mathrm{Pa})$; total nasal inspiratory flow $\left(\mathrm{L}^{\mathrm{s}} \mathrm{s}^{-1}\right)$ and the values of inspiratory flow resistance (KPA.L-1.s) were calculated as the mean of right and left nostrils of the test and the retest. Results were compared with pediatric reference values height-dependent reported in literature ${ }^{(14)}$.

According to Zapletal et al. the degree of nasal obstruction, based on rhinomanometry test values, was estimated as fraction of predicted values (p.v.) of rhinomanometric parameters: grade 1 corresponded to no obstruction (71-100\% of p.v.); grade 2 to mild obstruction (57-70\% of p.v.); grade 3 to moderate obstruction (43-56\% of p.v.); grade 4 to severe obstruction (29-42\% of p.v.); grade 5 to very severe obstruction (less than $29 \%$ of p.v.) ${ }^{(14)}$. Children were considered as correctly estimating their nasal obstruction when the grade of nasal patency corresponded to the 
Table 1. Nasal obstruction evaluated with objective and subjective methods at different pediatric age ranges. Significance was calculated with ANOVA.

$\begin{array}{lcccc} & \text { Total } & 6 \text { to } 9 \text { yr } & 9 \text { to } 12 \text { yr } & 12 \text { to } 14 \text { yr } \\ \begin{array}{l}\text { Nasal patency } \% \text { of } \\ \text { predicted values of } \\ \text { nasal flows) }\end{array} & 59.06 \pm 24.28 & 54.57 \pm 24.74 & 63.24 \pm 23.58 & 67.86 \pm 20.34 \\ & 33.59 \pm 20.87 & 34.23 \pm 20.67 & 34.5 \pm 23.01 & 30.14 \pm 18.13 \\ \text { NOSE score }(0-100) & & & >0.05\end{array}$

grade of NOSE score severity, overestimating when the grade of nasal patency was $<1$ when compared to the severity of the NOSE score, underestimating when the grade of nasal patency was $>1$ when compared to the severity of the NOSE score.

The study was approved by the Ethic Committee of "Sapienza" University of Rome and performed with the written informed consent of the parents of all children.

\section{Statistical analyses}

Statistical analyses were performed using SPSS (Statistical Package of Social Sciences, Chicago, IL, USA) software version 19. Descriptive statistics were performed expressing continuous data as means with SDs and categorical data were expressed by frequency and percentage. Comparisons were evaluated using a t-test and a chi-square test after assessing normality with the Kolmogorov-Smirnov test. Correlations between nasal patency and NOSE score were calculated with Pearson's correlation test. A p-value less than 0.05 was considered statistically significant.

\section{Results}

NOSE score and outcome of nasal patency

The mean NOSE score was $33.6 \pm 20.9$ and the mean Nasal patency at the rhinomanometric evaluation was 59.1 \pm 24 .3. No difference was detected for sex, atopic sensitization and NOSE score even though nasal patency was different among age ranges as shown in Table 1.

Global estimations and comparative depending on age and gender

Children in a different age range differently estimated their nasal obstruction as shown in Figure 1: in particular children between 6 and 9 years underestimated (43.7\%) and children $>12$ overestimated (34.7\%) their symptoms more frequently than children of other age groups $(p<0.001)$. Moreover, we found a significant increase in age from patients underestimating to patients correctly estimating and overestimating their nasal obstruction (Figure 2; p for the trend $<0.001$ ). Nose score and nasal patency in children underestimating, correctly estimating and overestimating nasal obstruction are shown in Figure 2. Concerning gender, in our sample, the percentage of fema- les correctly estimating was higher when compared to males ( $58.6 \%$ vs $39.3 \% ; p<0.002$ ) while males overestimated their nasal congestion more frequently than females ( $28 \%$ vs $13.8 \%$; $\mathrm{p}<0.002$ ) (Figure 3).

Correlation between NOSE score and nasal patency was statistically significant $(r-0.74 ; p<0.001)$ while it was not related to age.

\section{Discussion}

The relationship between objective and subjective methods has been debated for decades and the conclusion is far to be drawn. The interest for this issue is mainly due to the lack of knowledge on the mechanisms underlying the perception of nasal obstruction and the need to better understand when rhinomanometry should be regarded as a useful tool to decide if treatment is needed.

In the present study the relationship between objective and subjective methods was investigated performing anterior rhinomanometry and administrating a universally validated score such as NOSE score: in our sample of school aged children these methods showed a statistically significant correlation. This result

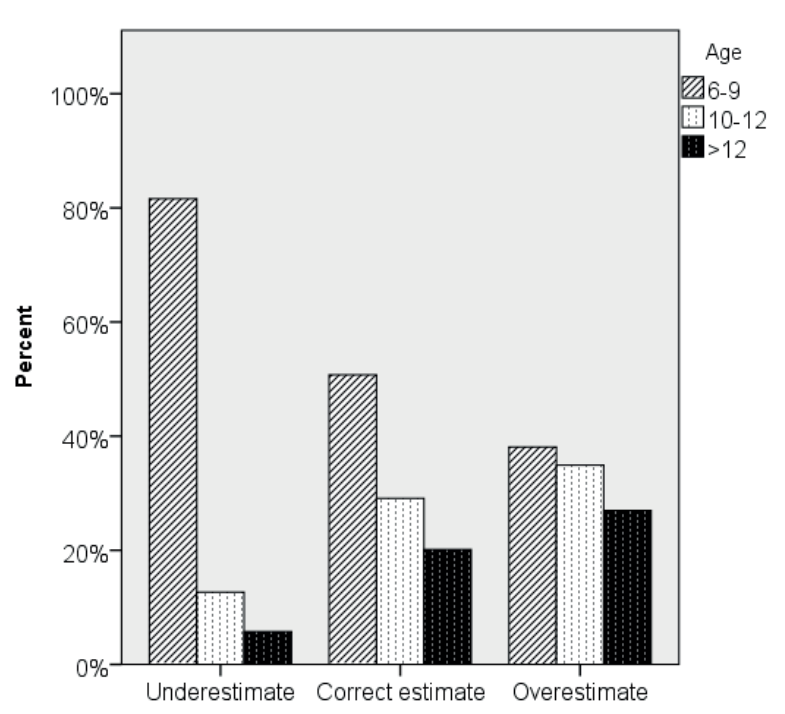

Figure 1. Percent of children at different pediatric age ranges underestimating, correctly estimating, overestimating. Differences between groups were calculated using a chi square test. ns = non significant. 
(A)

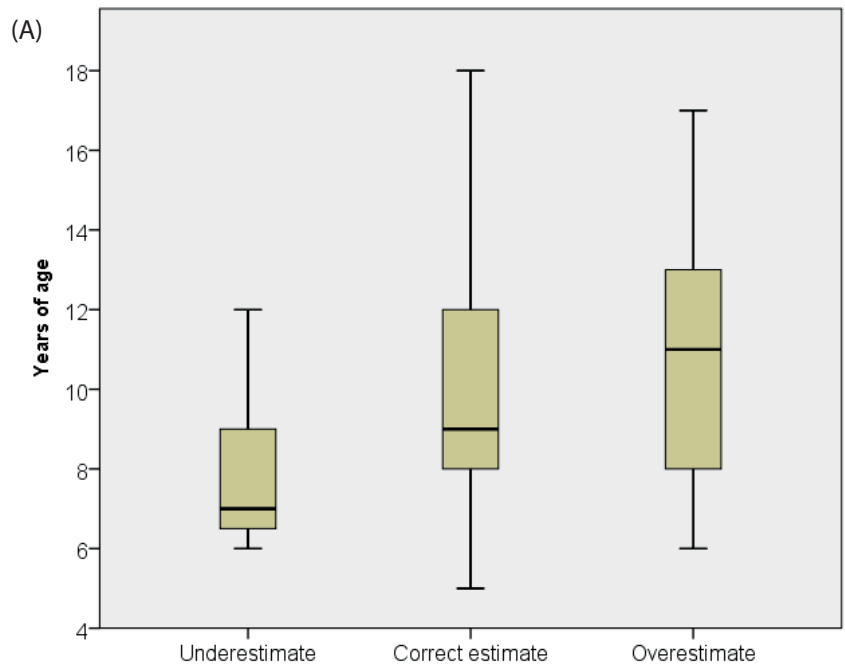

(B)
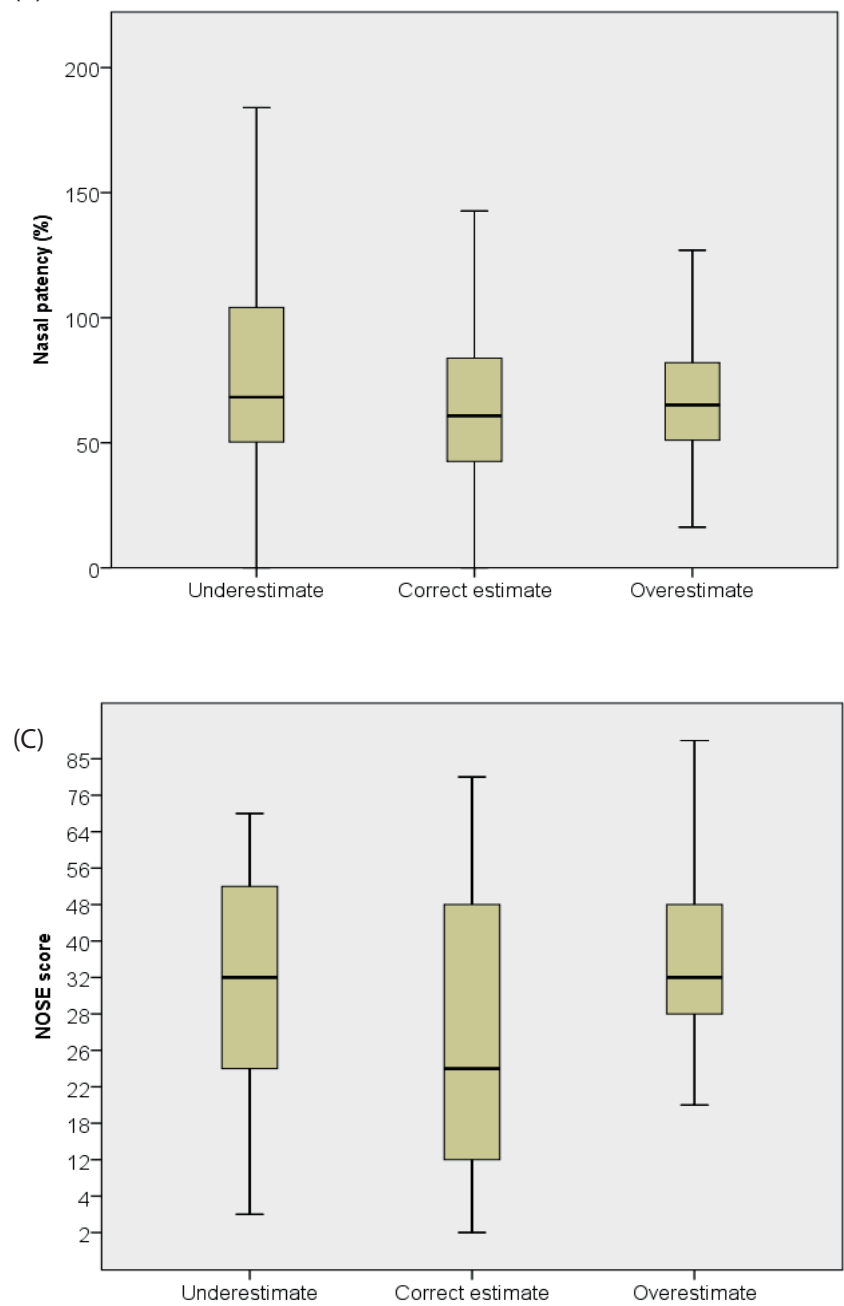

Figure 2. Years of age (A), Nasal patency (B) and NOSE score (C) in children underestimating, correctly estimating and overestimating. Differences were calculated using ANOVA with Bonferroni's post hoc test. Significance was indicated in the figures for the Bonferroni test. ns $=$ non significant. confirms that NOSE score approximately allow to quantify the sensation of nasal obstruction in children in line with the results of another previously published study ${ }^{(15)}$. In 1998, Szucks et al. found a significant correlation between the nasal patency and nasal airway resistance in patients with deviated nasal septum (16) and in 2006, Clarke et al. ${ }^{(17)}$ found the bigger the difference of the nasal airway resistance between nasal passages, the more likely that the passage subjectively indicated as more obstructed concurred with nasal airway resistance. Andre et al. ${ }^{(3)}$ concluded that the sensation of nasal airflow, at least under certain circumstances, can be entirely independent of any objectively measurable change in nasal resistance. Studies investigating this relationship in children are few. In 2010, Ciprandi et al. ${ }^{(18)}$ performed both anterior and posterior endoscopy and concluded that the employment of both Face Related Scale and Visual Analogue Scale allows, with good reliability, to objectify nasal obstruction in the absence of rhinomanometry.

The impact of the nasal cavity area on nasal airflow is difficult to foresee and their relationship should be regarded as really complex as described by the mathematic expression underlying the precise effects of nasal airway size on nasal airway resistance ${ }^{(19)}$. Although some studies found several significant associations between nasal cavity dimensions and nasal airflow, ${ }^{(20)}$ a possible limitation of the present study may be the lack of anatomical criteria considering that nasal resistence cannot perfectly reflect the cross sectional area of nasal cavity in dynamic aspects ${ }^{(21)}$. Of note, in our sample, children between 6 and 9 years of age often underestimate (43.7\%) and children $>12$ overestimate (34.7\%) their symptoms as confirmed by trend of age, NOSE score and nasal patency in patients underestimating, correctly estimating and overestimating. In 2006, Priftis et al. ${ }^{\left({ }^{8}\right)}$ suggested that children between 8-15 years on long term treatment for perennial rhinitis frequently under-report their symptom of nasal stuffiness. According to our knowledge, this the first study specifically focused on nasal obstruction at different pediatric age ranges with particular attention to the estimation of nasal patency.

Moreover, we found a difference in the subjective perception of nasal flow: females seem to estimate their nasal patency more accurately than males. This result should be analyzed considering that the activity of thermoreceptors and thermosensitive afferents may be influenced by many characteristics including gender ${ }^{(22)}$. However, establishing how much these differences can be attributed to learned behavior and cultural factors and to hormonal influences remains an important objective for future studies ${ }^{(23)}$.

\section{Conclusion}

The results of the present study underline that subjective and objective methods for the evaluation of nasal obstruction should be considered as complementary and then both 

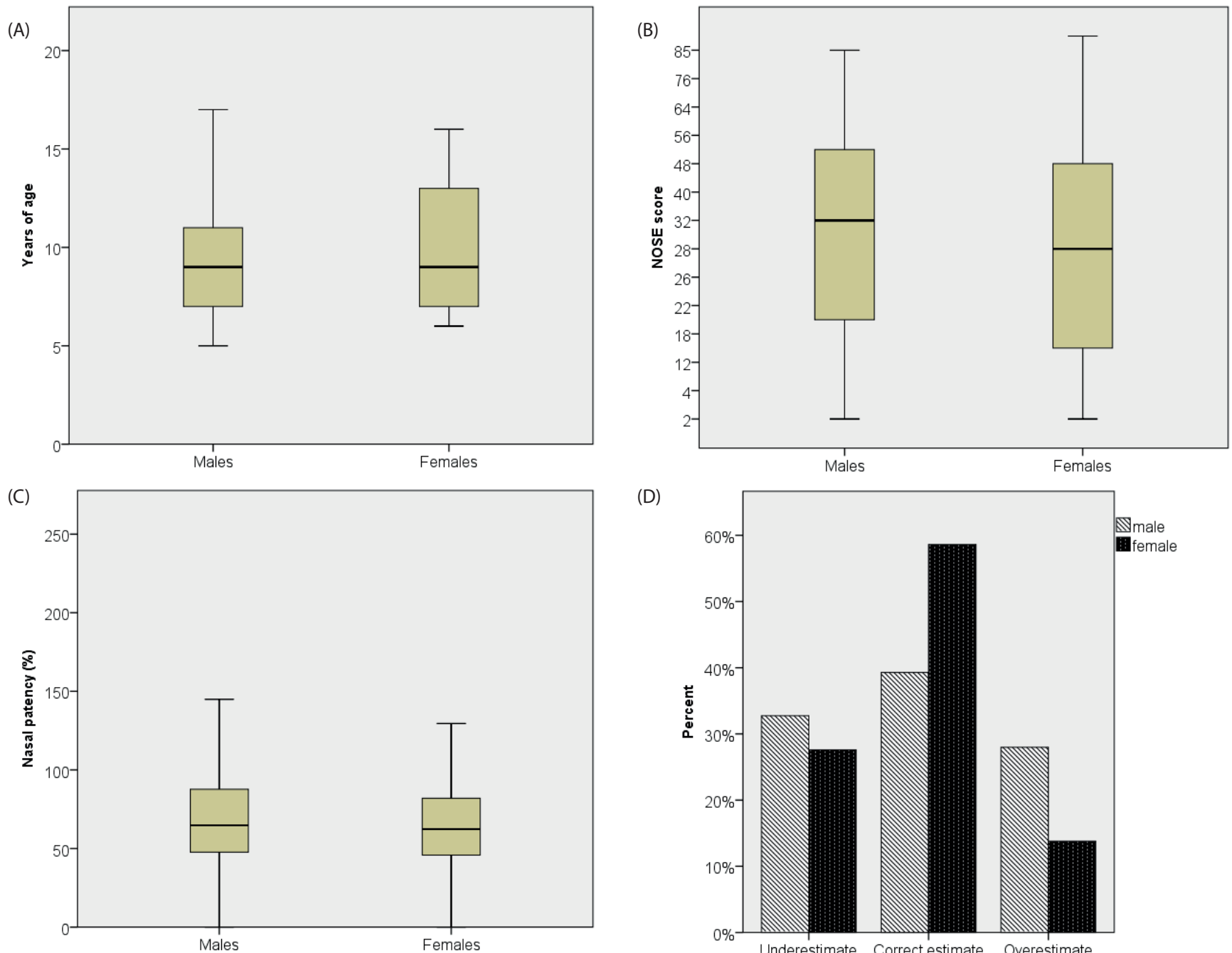

(D)

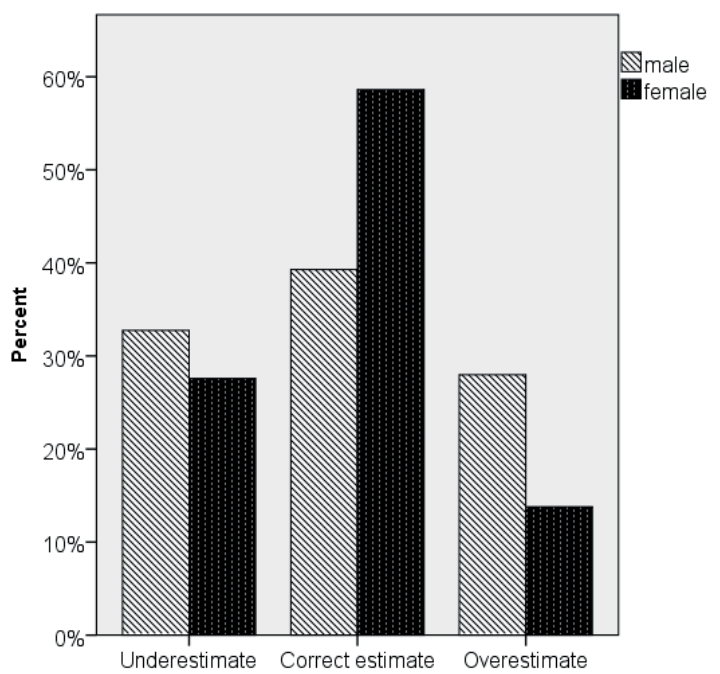

Figure 3. Years of age (A), NOSE score (B) and Nasal patency (C) and estimation of nasal patency in males and females (D). Differences were calculated using a t-test after assessing normality with a Kolmogorov-Smirnov test. Ns stands for non significant.

performed since they investigate different aspects of nasal obstruction. In this perspective, considering the evidence of the relationship between systemic inflammation and rhinitis ${ }^{(2)}$, especially in children aged between 6 and 9 years an objective measurement of nasal patency should be performed to better define the correct therapeutic approach.

\section{Authorship contribution}

Study concept and design: FO, AMZ. Acquisition of data:TV, AR,
GT, GDC, LI. Analysis and interpretation of data: FO, MD, TV, AR, GT, GDC, LI, AMZ. Drafting of the manuscript: FO, AMZ. Critical revision of the manuscript for important intellectual content: MD, AMZ. Statistical analysis: FO. Study supervision: MD, AMZ.

\section{Conflict of interest}

The authors have no conflict of interest related with this manuscript.

\section{References}

1. Meltzer EO, Blaiss MS, Derebery J, et al. Burden of allergic rhinitis: results from the pediatric allergies in America survey. J Allergy Clin Immunol 2009; 124: S43-70.

2. Zicari AM, Occasi F, Cesoni Marcelli C, et al. Assessing the relationship between serum resistin and nasal obstruction in children with allergic rhinitis. Am J Rhinol Allergy
2013; 27: 127-30.

3. André RF, Vuyk HD, Ahmed A, et al. Correlation between subjective and objective evaluation of the nasal airway. A systematic review of the highest level of evidence. Clin Otolaryngol 2009; 34: 518-25.

4. Yepes-Nuñez JJ, Bartra J, Muñoz-Cano R, et al. Assessment of nasal obstruction: correlation between subjective and objective techniques. Allergol Immunopathol 2013; 41: 397-401.

5. Sozansky J, Houser SM. The physiological mechanism for sensing nasal airflow: a literature review. Int Forum Allergy Rhinol 2014; 4: 834-8.

6. Keh SM, Facer P, Simpson KD, Sandhu G, Saleh HA, Anand P. Increased nerve fiber expression of sensory sodium channels 
Nav1.7, Nav1.8, And Nav1.9 in rhinitis. Laryngoscope 2008; 118: 573-9.

7. Thorstensen WM, Sue-Chu M, Bugten V, Cvancarova M, Steinsvåg SK. The determining factors of peak nasal inspiratory flow and perception of nasal airflow in asthmatics. Rhinology. 2014; 52: 348-54

8. Priftis KN, Drigopoulos K, Sakalidou A, et al. Subjective and objective nasal obstruction assessment in children with chronic rhinitis. Int J Pediatr Otorhinolaryngol 2006; 70: 501-5.

9. Bousquet J, Schünemann HJ, Samolinski $B$, et al. Allergic Rhinitis and its Impact on Asthma (ARIA): achievements in 10 years and future needs. J Allergy Clin Immunol 2012; 130: 1049-62.

10. Jáuregui I, Dávila I, Sastre J, et al. Validation of ARIA (Allergic Rhinitis and its Impact on Asthma) classification in a pediatric population: the PEDRIAL study. Pediatr Allergy Immunol 2011; 22: 388-92.

11. Mozzanica F, Urbani E, Atac M, et al. Reliability and validity of the Italian nose obstruction symptom evaluation (I-NOSE) scale. Eur Arch Otorhinolaryngol 2013; 270: 3087-94.

12. Lipan MJ, Most SP. Development of a severity classification system for subjective nasa obstruction. JAMA Facial Plast Surg 2013; 15: 358-61.

13. Clement PA. Committee report on stand- ardization of rhinomanometry. Rhinology 1984; 22: 151-5

14. Zapletal A, Chalupovà J. Nasal airflow and resistance measured by active anterior rhinomanometry in healthy children and adolescents. Pediatr Pulmonol 2002; 33; 174-180.

15. Zicari AM, Occasi F, Giulia M, et al. Intranasal budesonide in children affected by persistent allergic rhinitis and its effect on nasal patency and Nasal Obstruction Symptom Evaluation (NOSE) score. Curr Med Res Opin $2015 ; 31$ : 391-6.

16. Szücs E, Clement PA. Acoustic rhinometry and rhinomanometry in the evaluation of nasal patency of patients with nasal septa deviation. Am J Rhinol 1998; 12: 345-52.

17. Clarke JD, Hopkins ML, Eccles R. How good are patients at determining which side of the nose is more obstructed? A study on the limits of discrimination of the subjective assessment of unilateral nasal obstruction. Am J Rhinol 2006; 20: 20-4.

18. Ciprandi G, Tosca MA, Signori A, Ameli F. Comparison between symptoms and endoscopy in children with nasal obstruction. Int J Pediatr Otorhinolaryngol. 2010; 74: $1405-8$.

19. Warren DW, Hairfield WM, Seaton DL, Hinton VA. The relationship between nasal airway cross-sectional area and nasal resistance. Am J Orthod Dentofacial Orthop
1987: 92: 390-5

20. Kjaergaard T, Cvancarova M, Steinsvåg SK Relation of nasal air flow to nasal cavity dimensions. Arch Otolaryngol Head Neck Surg 2009; 135: 565-70.

21. Kim CS, Moon BK, Jung DH, Min YG. Correlation between nasal obstruction symptoms and objective parameters of acoustic rhinometry and rhinomanometry. Auris Nasus Larynx 1998; 25: 45-8.

22. Schepers RJ, Ringkamp M. Thermoreceptors and thermosensitive afferents. Neurosci Biobehav Rev 2010; 34: 177-84.

23. Doty RL, Cameron EL. Sex differences and reproductive hormone influences on human odor perception. Physiol Behav 2009; 97: 213-28.

Francesca Occasi

Department of Pediatrics

university "La Sapienza"

Rome

Italy

Tel: $+33-32547775$

E-mail: occasi.f@gmail.com 